\title{
ON AFFINE NON-NEGATIVE MATRIX FACTORIZATION
}

\author{
Hans Laurberg ${ }^{1}$ and Lars Kai Hansen ${ }^{2}$ \\ ${ }^{1}$ Department of of Electronic Systems, Aalborg University, \\ Fredrik Bajers Vej 7A-3, DK-9220 Aalborg, Denmark, \\ ${ }^{2}$ Informatics and Mathematical Modelling, Technical University \\ of Denmark B321, DK-4000 Lyngby, Denmark, \\ emails: $\{$ hla $\} @$ kom.aau.dk, $\{1 \mathrm{kh}\} @$ imm.dtu.dk
}

\begin{abstract}
We generalize the non-negative matrix factorization (NMF) generative model to incorporate an explicit offset. Multiplicative estimation algorithms are provided for the resulting sparse affine NMF model. We show that the affine model has improved uniqueness properties and leads to more accurate identification of mixing and sources.
\end{abstract}

Index Terms - Non-negative matrix factorization, NMF, BSS, Sparse NMF

\section{INTRODUCTION}

Non-negative matrix factorization (NMF) has become a popular tool for data analysis. An often stated reason for NMF is that it leads to 'parts based' representations, hence, facilitates data analytic interpretation. However, uniqueness is important for the parts based representations to be meaningful. The NMF generative model is based on linear mixing of positive sources by positive coefficients. The positive sources may have offsets which can lead to non-uniqueness, we therefore here propose a model based on affine mixing, i.e., mixing with an offset. The NMF learning algorithm is straightforwardly generalized to handle the augmented model. We show that the affine model indeed has improved uniqueness properties and thus leads to more accurate identification of mixing and sources.

NMF algorithms are used to factorize a nonnegative matrix $V \in$ $\mathbb{R}^{N \times M}$ in two nonnegative matrices $W \in \mathbb{R}^{N \times D}$ and $H \in \mathbb{R}^{D \times M}$

$$
V \approx R=W H ; \quad V_{i, j} \approx R_{i, j}={ }_{d=1}^{D} W_{i, d} H_{d, j}
$$

Following the seminal papers by Lee and Seung [?, ?], a least squares or a Kullback-Leibler inspired cost are used. Our observations in this paper can be applied to both. For simplicity we will concentrate on the Euclidian cost in the following,

$$
E(W, H)=\|V-W H\|_{F}^{2},
$$

where $\|\cdot\|_{F}$ is the Frobenius norm. Lee and Seung[?] have shown that the following update rule will decrease $E(W, H)$ :

$$
\begin{gathered}
H \leftarrow H \otimes \frac{W^{T} V}{W^{T} R} \\
W \leftarrow W \otimes \frac{V H^{T}}{R H^{T}},
\end{gathered}
$$

This research was supported by the Intelligent Sound project, Danish Technical Research Council grant no. 26-02-0092. where $\otimes$ and $\frac{(\cdot)}{(\cdot)}$ are element wise multiplication and division. This update rule is used as a reference and is shown in panel (B) of figures $1,3,4,5$ and 6 .

\section{SPARSE NMF}

Hojer [?] introduced sparse NMF and Eggert[?] proposed the following cost function where only the normalized version of $W$ has impact on the cost:

$$
\begin{aligned}
E(W, H) & =\frac{1}{2} V-\bar{W} H_{F}^{2}+\lambda \mathbf{1}^{T} H \mathbf{1} \\
\bar{W}_{n} & =\frac{W_{n}}{\left\|W_{n}\right\|}, n \in\{1, \ldots, N\}
\end{aligned}
$$

where $W_{n}$ is the n'th column vectorin $W$ and $\mathbf{1}$ is a column vector where all elements are one. The length of $\mathbf{1}$ can be deduced by the context. The scalar $\lambda$ is a positive parameter that controls the tradeoff between sparseness of $H$ and approximation of $V$ by the product of $W, H$. Eggert[?] argues for using the following multiplicative update:

$$
\begin{aligned}
H & \leftarrow H \otimes \frac{\bar{W}^{T} V}{\bar{W}^{T} R+\lambda} \\
W_{n} & \leftarrow \bar{W}_{n} \otimes \frac{{ }_{m=1}^{M} H_{m, n}\left(V_{n}+\bar{W}_{n}\left(R_{m}\right)^{T} \bar{W}_{n}\right)}{{ }_{m=1}^{M} H_{m, n}\left(R_{n}+\bar{W}_{n}\left(V_{m}\right)^{T} \bar{W}_{n}\right)}
\end{aligned}
$$

These update rules are used in panel (C) of figures 1, 3, 4, 5 and 6.

The normalization of $W$ and the sparse nature of $H$ critically constrains the solution and can improve uniqueness and lead to more accurate estimates. However, the constraints may not be consistent with the form of the mixing process and the statistics of the source signals $H$. In particular offsets in one or more rows of $V$ will counteract the sparse model. If the generative model incorporates additive noise it is not clear that simple subtraction of the minimal value of each row in $V$ will lead to a correct recovery of the generating $W, H$. If the noise is, e.g., Gaussian, $V$ can be negative in the native representation, hence, one cannot estimate the 'true' offset.

\subsection{Affine Sparse NMF}

The above sparse NMF methods do not handle offsets, however, it is incorporated as follows with $W_{0} \in \mathbb{R}^{N \times 1}$

$$
V \approx R=W H+W_{0} \mathbf{1}^{T}
$$


Fig. 1. Simulated data where $V \in \mathbb{R}^{2 \times 2000}$ is generated according to Equation 9. Each column of $V$ is plotted as a dot. In (A) the generating $W$ and $W_{0}$ are shown. In (B) and (C) the standard NMF and sparse NMF each find three vectors that can describe the data. Both algorithms find one vector that is a linear combination of the true $W_{0}$ and $W_{1}$ and finds two vectors that are very close to the true $W_{0}$. In (D) the 'Affine sparse NMF' method correctly estimates the structure of the $W$ matrix.

Using this augmented signal model the sparse cost function in Equation 5 becomes

$$
E\left(W, H, W_{0}\right)=\frac{1}{2} \quad V-\bar{W} H-W_{0} \mathbf{1}_{F}^{T}{ }_{F}^{2}+\lambda \mathbf{1}^{T} H \mathbf{1}
$$

Using the same arguments as Eggert[?] the update rule for $W$ and $H$ remains as given in Equation 7 and 8 using the new definition of $R$. Since $W_{0}$ is not normalized it is updated using the standart NMF update rule (Equation 4) and not the sparse update rule (Equation 8):

$$
W_{0} \leftarrow W_{0} \otimes \frac{\mathbf{1}^{T} V}{\mathbf{1}^{T} R}
$$

The affine sparse NMF results are shown in panel (D) of figures 1 , $3,4,5$ and 6

\section{RESULTS}

How does the augmented sparse affine NMF model data? To answer this question we first visualize synthetic data as generated by the proposed model, and we show existing methods fail to reconstruct the correct parameters of the generative model. We then go on to show that two commonly used data sets have the characteristics of the proposed model and that the proposed algorithm performs better than the existing algorithms on the data. In order to get a 'fair' comparison the standard NMF and sparse NMF both have one column more than the sparse affine NMF method. This ensures that the maximum rank of $R$ is the same for all methods.
Fig. 2. The variation of the relative least squares error of the NMF reconstruction of $W$. The error is plotted as a function of the amount of data $(M)$. The simulated data was generated using $D=10$ components and an off set. The 'zero offset' methods are based on the simple heuristic that data is first preprocessed to have minimum value zero in each row.

Simulated Data. In Figure 1 there are $M=2000$ elements in $V$. The data is generated as in Equation 9. The elements of $R$ are exponentially distributed. The true $W$ vectors and the column vectors of $V$ are shown in Figure 1 panel (A). Figure 1 (B-D) shows the three different algorithms estimate of $W$. The standard NMF (B) finds $W$ such that the data is in the positive span of $W$. The $W$ estimated by the sparse NMF algorithm (C) also spans data but the column vectors of $W$ point more directly towards data. Although these methods estimated $W$ can reproduce $V$, they do not find the correct structure $(W)$. The proposed method (D) finds a $W$ that is close to the true $W$.

A quantitative evaluation of the different algorithms' estimate is presented in Figure 2. Data is generated as in Equation 9 where the elements of $W$ and $W_{0}$ are uniform i.i.d. The elements of $H$ are first generated as exponential i.i.d. samples and then each column is normalized to unit sum. In this way the elements in $H$ describe how much each column vector of $W$ contribute towards $V$. In all simulations $N=100, D=10$. We have run the simulation with different amounts of data exampels (column in $V$ ) $M$. In the evaluation $V$ is analysed as 11(=10+1) outer product ${ }_{d=0}^{D} V^{(d)}=V$, where $V_{i, j}^{(d)}=W_{i, d} H_{d, j}$. The error in the figure is the relative least squares error of the $V^{(d)}$ estimate for each data set size

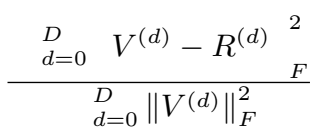

For completeness we have here included in the performance evaluation a modification of the standard method in which data is first 
Fig. 3. Subset of A: The Swimmer database B: Basis pictures using standard NMF. C: Basis pictures using sparse NMF. D: Basis pictures using sparse affine NMF.

subtracted with constant offsets to achieve zero minimum value in each of the $N$ variables of $V$. The simulation shows that the standard NMF and the sparse NMFs do not find the true $W$ and $H$. The constant offset subtraction improves the performans but is outperformed by the sparse affine NMF succeeds. Notis that the two latter methods is favoured by knowing that $\left(H^{T}\right)_{0}=\mathbf{1}$.

The Swimmer Database. The "Swimmer Database" was introduced by Donoho and Stodden [?] to discuss the uniqueness issue we have adressed in this presentation. The point was that even if NMF can represent $V$ it may not necessarily find the right $W$. The database consist of $256(32 \times 32$ pixel) black-and-white pictures of a 'stick-man' with 4 limbs that can be in one of 4 positions. All pictures have a 'torso' that represent an offset as discussed in this paper. The pictures in the dataset can be constructed by $17(=4 \times 4+1)$ non-overlapping basis pictures. In Figure 3 (A) examples from the database are shown. The algorithms described in section 2 are tested on the data set and a subset of the 17 basis pictures are shown in Figure 3(B-D). Only the proposed method is able to find the 17 nonoverlapping basis pictures, the standard NMF and Sparse NMF all let the torso be a part of all basis pictures. The Swimmer simulation are further analyzed in Figure 4. The $1024(=32 \times 32)$ dimensional column vectors in $V$ and $W$ are mapped onto a two dimensional subspace to show that the structure of the swimmer database is in fact equivalent to that of Figure 1. In the plot it is seen that only the affine sparse NMF finds the true basis vectors.

Business Card Data Set. Our final example is based on a set of business card images of faculty of Aalborg University's Department of Electronic Systems. The photographer has manually centered and scaled the pictures. The pictures are scaled to $30 \times 40$ pixel and the color map is chosen such that white is zero and black is maximum. An 'AAU watermark' logo has been added to all pictures in the database. A subset of the pictures are shown in Figure 5(A) and
Fig. 4. A two dimensional subspace of the column vectors in $V$ (dots) and $W$ (vectors) are shown for the Swimmer database. The ' $\mathrm{x}$ axis' is a picture which is zero in the upper part and uniform random values in the lower part. The 'y-axis' is constructed the same way but with the zeros in the lower part.

a subset of the 25 basis pictures estimated by the three algorithms is shown in Figure 5(B-D). In this simulation the sparse affine NMF algorithm estimates more sparse basis pictures and most basis pictures describe one physical object only.

A two dimensional subspace (axes formed by an picture with 'hair' and an picture with the AAU-logo) of the images in Figure 5 are shown in Figure 6. As above we find that none of the standard NMF's nor sparse NMF basis vectors describe the AAU logo without also capturing 'hair'. The basis pictures for the proposed method however are found close to the axes meaning that they either capture hair or the AAU' logo.

\section{DISCUSSION AND CONCLUSION}

Non-negative matrix factorization is widely applied because of the ability to create 'parts based' representations, hence, facilitating model interpretation. However, uniqueness is important for the parts based representations to be meaningful. Lack of uniqueness can happen in several ways, e.g., due to an offset vector $W_{0}$ as discussed here. Another mechanism resulting in lack of uniqueness is if the support of the process creating a row of $H$ does not include $H=0$, i.e., if there is an offset in the row variable of $H$. The $H_{0}$ offset can be seen as a $W_{0}$ offset with the constraint that $W_{0}$ is in the positive span of the column vectors in $W$ :

$$
R=W\left(H+H_{0} \mathbf{1}^{T}\right)=W H+W_{0} \mathbf{1}^{T}, W_{0}=W H_{0}
$$

Hence, the $H$ offset issue is a special case of the model we have discussed here: If the resulting $W_{0}$ is in the positive span of the columns of $W$, they can be interpreted as $H$ offsets 
Fig. 5. (A): Subset of the Picture database with 197 pictures (B D): A subset of the basis pictures using standard NMF, sparse NMF and sparse affine NMF. The standard NMF makes very noisy basis pictures. The sparse NMF produce basis pictures where the 'AAU watermark' is visible in around $50 \%$ of the pictures, and in addition a lot of the pictures do not represent a single part of the picture. The sparse affine NMF has only one picture with the watermark $\left(W_{0}\right)$ and most pictures represent only one part of the picture.

In this work we have defined the augmented non-negative linear mixing model - the sparse affine NMF. While the most obvious solution to an offset may appear to preprocess data such that the minimum value is zero, we have shown that there is straightforward data driven way of optimizing such offset. We have presented three case stories in which the new sparse affine NMF algorithm outperforms the standard algorithms in the estimation of the underlying structure of the data.
Fig. 6. The business card images plotted in two dimensions to show that data and solutions have pattern like the ones in Figure 1. The $\mathrm{x}$-axis is the an image of the AAU logo, and the $\mathrm{y}$-axis is an image vector capturing the 'hair' region. 